\title{
A UTILIZAÇÃO DE GRUPOS DE ESTUDOS PARA O APRENDIZADO NA GRADUAÇÃO
}

José Antônio Aravena-Reyes - jose.aravena@ufjf.edu.br

Mariana Larissa Antunes da Costa - mariana.antunes@engenharia.ufjf.br

Michele Nogueira Reis de Paula - michele.nogueira@engenharia.ufjf.br

Universidade Federal de Juiz de Fora, Faculdade de Engenharia

Rua José Lourenço Kelmer, s/n - Campus Universitário - Bairro São Pedro

36.036-900 - Juiz de Fora - Minas Gerais

Resumo: As novas diretrizes curriculares advogam por uma maior formação de soft skill nos cursos de Engenharia e, dentre as competências a serem desenvolvidas, a capacidade de trabalho em equipe, a criatividade e a crítica são altamente recomendadas. Ao encontro dessa diretriz, na Universidade Federal de Juiz de Fora existe uma normativa que promove atividades docentes fora da sala de aula, privilegiando espaços de aprendizagem mais eficientes que tornem o estudante o agente ativo da sua aprendizagem. $O$ trabalho em grupo pode, portanto, ser um bom modelo para desenvolver as competências desejadas. Este estudo apresenta e discute a metodologia do grupo de estudos que adotam uma perspectiva de maior autonomia dos estudantes na organização das atividades. Primeiramente é apresentado o contexto e marco geral da proposta, para depois elencar os resultados obtidos mediante essa prática metodológica. Finalmente os resultados de uma pesquisa qualitativa são apresentados para descrever a alta receptividade que esta abordagem tem entre alunos e professores.

Palavras-chave: Metodologia de ensino, Grupos de Estudo, Educação.

\section{INTRODUÇÃO}

A Educação em Engenharia é um processo muito particular que exige tanto a obtenção de um perfil do egresso, isto é, as competências que devem ser desenvolvidas nele, quanto uma abordagem metodológica que seja eficiente no aprendizado dessas. Em geral, perfil e forma metodológica para o ensino são instruídas pelas chamadas DCNs - Diretrizes Curriculares Nacionais para o Curso de Graduação em Engenharia (BRASIL, 2019). A recente normativa coloca em pauta aquilo que se chama soft skills (DIXON et al., 2010), um conjunto de competências profissionais direcionadas a aprimorar a perspectiva social e gerencial do egresso. Entre essas competências, o trabalho em equipe, o desenvolvimento da crítica e a da criatividade são algumas das dimensões abordadas.

Por outro lado, desde algum tempo, existem instrumentos institucionais que promovem espaços alternativos à forte formação mediante aula expositivas, que visam tornar mais eficiente o processo de aprendizagem do aluno. Em geral, a flexibilização curricular busca criar outros ambientes de aprendizagem, o que acarreta também, o uso de outras metodologias de ensino. Entre as alternativas que existem, as chamadas metodologias ativas (MORÁN, 2015) resultam de particular interesse uma vez que promovem a participação ativa do estudante no processo de aprendizagem, motivando-o a atingir espaços de autonomia, compreensão e engajamento que a 
tradicional abordagem da aula expositiva não proporciona. No caso abordado neste estudo, se argumenta a favor dos chamados grupos de estudos (UFJF, 2016), grupos onde os estudantes, voluntariamente, se envolvem em temas do interesse deles mesmos e que geralmente, se encontram fora do processo formativo que consta nas disciplinas. O grupo, como organização estudantil, é um espaço de aprendizagem em que seus participantes são alocados para a construção coletiva e a leitura crítica da realidade, que cria uma interdependência no compartilhamento de tarefas e passa a aprender a planejar e colaborar (MAXIMINO, 2015).

Os grupos também podem atuar como camisas de força, que obrigam o estudante a se adaptar a uma cultura interna de valores, "sujeitando" os participantes neles ou dando ampla liberdade para que construam um grupo como um autêntico onde eles se tornam sujeitos ativos da aprendizagem (MYERS, 2014). Nesse sentido, a forma de abordar o trabalho de um grupo de estudo pode ser, indicando uma sequência rigorosa de textos e temas a serem foco do estudo em grupo ou, como se optou nesta experiência, com total liberdade para o aluno escolher os temas, organizar as atividades e definir os meios de avaliação.

O objetivo deste ensaio teórico reside em apresentar e discutir a metodologia do Grupo de Estudos como prática de aprendizagem na graduação. Inicialmente é explicado o contexto onde se desenvolvem as atividades, para depois descrever a sua abordagem metodológica. As atividades mais relevantes de dois grupos de estudo constituídos a partir da perspectiva defendida aqui são apresentados para finalmente apresentar os resultados de uma pesquisa feita como os integrantes discentes e docentes dos grupos para extrair a opinião deles sobre a experiência vivenciada.

\section{Grupos de Estudos}

Muito ouve-se dizer sobre Grupos de Pesquisas, os quais são institucionalizados dentro das universidades como método de ensino, aprendizado e flexibilização curricular, orientado por um docente, com objetivos fixos e predefinidos em seu contexto. Já o Grupo de Estudos é composto por um orientador, que tem o papel de mediação dentro do núcleo, e os estudantes interessados na área geral de estudo, sendo que o objeto de estudo é mutável. Nele, os estudantes são responsáveis pela regência do grupo, ao contrário do que se ocorre em grupos de pesquisa, em que essa responsabilidade cabe ao orientador.

O modelo de aprendizagem aqui defendido é a metodologia do Grupo de Estudos como prática de aprendizagem na graduação. Esta metodologia de aprendizado tem como objetivo propiciar aos alunos o aprendizado do convívio social, desenvolver o interesse em áreas específicas, bem como a liderança e o trabalho em equipe. A metodologia ativa é utilizada nesse tipo de grupo, com o estudante como sujeito, o que faz com que haja o desenvolvimento do pensamento crítico e reflexivo do discente, desenvolvendo autonomia, responsabilidade e a capacidade de analisar e solucionar questionamentos (SOBRAL; CAMPOS, 2012).

O grupo de estudos aqui considerado se estrutura com uma distribuição horizontal das responsabilidades entre os estudantes e o orientador, sendo que este, mesmo com sua experiência de docência, aprende junto com os discentes. Mesmo a grande área sendo única, suas ramificações são amplas, instigando os participantes à busca por informações de seu interesse.

A organização das atividades do Grupo de Estudos se baseia em definir um tema central e, juntamente com os estudantes, descobrir ramificações dele que sejam de interesse de estudo as quais se exploram, em reuniões semanais, que permitem acrescentar as perspectivas que os alunos possuem para tratar o tema. O processo se inicia ao começo de cada semestre, momento em que é realizado um brainstorming para definir os possíveis casos de estudo do semestre. Os assuntos com maior interesse são discutidos e cada estudante fica responsável pela busca de 
informações sobre o tema para o grupo. A dinâmica natural do grupo é conduzida pelos interesses dos alunos, deixando o professor como um mediador e estimulador da inteligência coletiva (LEVY, 1998). À medida que o semestre progride, tarefas são definidas para cada membro do grupo e resultados compartilhados encontro após encontro.

\section{O GRUPO DE ESTUDOS EM GRANDES OBRAS DE INFRAESTRUTURA}

O GEInfra, Grupo de Estudos de Grandes Obras de Infraestrutura, da Faculdade de Engenharia da Universidade Federal de Juiz de Fora (UFJF), teve início em 2016 com o objetivo de despertar a curiosidade dos alunos e alunas dos cursos de Engenharia para assuntos relacionados ao projeto e gestão de objetos ou obras de infraestrutura no Brasil. É orientado pelo Prof. DSc. José Aravena-Reyes e foi criado a partir da iniciativa de alunos para suprir conteúdos pouco ou não abordados na faculdade.

O GEInfra levanta dados e, através de discussões e referências técnicas, analisa problemas e outras questões no âmbito da infraestrutura e sociedade, como nos casos da BR-440, do Projeto Village Marie, no Haiti e a Barragem de Casa de Pedra, em Congonhas - MG. Além disso, é feito também o estudo de literaturas com temas inerentes à engenharia.

Como tema relevante para o profissional de engenharia, o grupo visa suprir a pouca atenção que recebe a temática desde a perspectiva sistêmica, isto é, considerando o conjunto de variáveis técnicas, psico-sócio-políticas e ambientais das grandes obras e empreendimentos da sociedade. O grupo utiliza como ponto de partida casos reais como objetos de aplicação para, sobre esses, articular estudos que vão desde a revisão de literatura técnica até organização e realização de eventos e visitas técnicas, incluindo rodas de discussões com comunidades e responsáveis pelos casos estudados.

As atividades do grupo envolvem reuniões semanais de uma hora em horário a combinar, na qual são discutidos os temas e os procedimentos a serem desenvolvidos durante cada semestre. $\mathrm{O}$ núcleo abre a cada semestre um pequeno número de vagas de modo a ter um número constante de membros que varia entre 4 e 6 . As temáticas são abordadas mediantes casos práticos que exigem o levantamento de informações, discussões técnicas, de política, gestão e eventualmente, convites a especialistas para abordar temas específicos.

\subsection{Estudo sobre a BR-440}

O caso da BR 440 teve como motivação o interesse do grupo em entender uma obra de grande magnitude e impacto com proximidade do ambiente da Universidade Federal de Juiz de Fora. Com diversas desapropriações sendo noticiadas e por Juiz de Fora já apresentar altos índices de déficit habitacional, voltaram-se as atenções para o caso.

Dessa forma, em 2016 foi realizado um levantamento de informações sobre o andamento do projeto da BR 440 que teve início na década de 80 e, então listados todos os problemas que eram de conhecimento do grupo, como a desapropriação de moradias e a falta de postura da Prefeitura e da empresa licitada para a construção na época.

Pelas dificuldades encontradas no estudo, foram convidados para participar das discussões alunos de mestrado do Programa de Pós Graduação em Ambiente Construído da (UFJF), o Professor DSc Cézar Henrique Barra Rocha do departamento de Transportes e Geotecnia da UFJF e a advogada Ilva Facio Netto Lasmar - pessoas já envolvidas em outros estudos dessa situação. 


\subsection{Estudo do Projeto Village Marie}

Em relação ao Projeto Village Marie, no Haiti, o grupo teve conhecimento sobre o tema após um membro sugerir para discussão o livro "(Re)construindo um sonho", escrito pelo pesquisador haitiano Jac-ssone Alerte, formado na Universidade Federal do Rio de Janeiro. O livro levantou discussões de como fomentar as construções habitacionais sustentáveis em comunidades carentes e atuar como um agente transformador da realidade, já que se trata de um projeto desenvolvido pelo engenheiro de reconstruir o Vilarejo Don de L'amitié no distrito de Grand' Anse, no Haiti.

O projeto se baseia em Solução Habitacional Simples (ALERTE, 2017) o qual foi concebido com a filosofia de reunir conhecimentos básicos que podem ser úteis na reconstrução de unidades habitacionais e equipamentos coletivos básicos, em regime de mutirão, a partir do emprego de tecnologias de baixo custo. Na referida situação, a tecnologia utilizada é a do tijolo ecológico solo cimento, que utiliza o solo do próprio local. Com o estudo deste livro, o grupo conseguiu, por meio da Universidade Federal de Juiz de Fora, trazer o autor para uma palestra durante o evento "Para Além da Catástrofe", o qual será anunciado posteriormente.

\subsection{Estudo sobre a Barragem de Casa de Pedra}

A Barragem de Casa de Pedra, maior barragem de terra em região municipal, com volume de 50 milhões $\mathrm{m}^{3}$, com altura aproximada de $76 \mathrm{~m}$ e Classe 6 , a mais alta em categoria de risco e de dano potencial associados de uma grande mineradora da região do Quadrilátero Ferrífero, localizada em Congonhas (MG), foi o último tema estudado pelo grupo em 2019. Esse assunto chegou ao grupo em junho de 2018, como atividade para o próximo semestre letivo, trazido por uma de suas integrantes que era moradora da cidade.

O grupo buscou informações sobre o assunto em literaturas e leis sobre o assunto, com a empresa proprietária, com um líder comunitário da cidade e com docentes da UFJF especialistas em geotecnia. Ao entrar em contato com a empresa, conseguiu-se uma visita técnica ao local. Porém, como não existe verba destinada a Grupos de Estudos pela Faculdade de Engenharia a qual o núcleo está vinculado, todos os valores gastos pela viagem (a cidade é localizada a 180 $\mathrm{km}$ de Juiz de Fora) tiveram que ser divididos entre os integrantes.

\subsection{Evento Para Além da Catástrofe}

Após o desastre ambiental de Brumadinho, ocorrido em Janeiro de 2019, o grupo juntouse ao Ramo Estudantil IEEE UFJF, ao PET Civil UFJF e ao Engenheiros sem Fronteiras núcleo Juiz de Fora para a realização de um evento nomeado "Para Além da Catástrofe", que buscou trazer conhecimentos sobre barragens de resíduos de mineração e outras finalidades para os mesmos.

Para tal, foram convidados os professores Dsc. Romero César Gomes e Dsc. Wanna Carvalho Fontes, da Universidade Federal de Ouro Preto, a docente Dsc. Júlia Righi de Almeida do departamento de Transportes e Geotecnia da UFJF, do líder comunitário congonhense Sandoval de Souza Pinto Filho e o já apresentado Jac-ssone Alerte. Para a realização de tal, houve apoio do Centro Regional de Inovação e Transferência de Tecnologia (CRITT) e da Faculdade de Engenharia da UFJF. 


\section{O GRUPO DE ESTUDOS E PRÁTICAS EM BIM}

De acordo com o site oficial do Grupo de Estudos e Práticas em BIM (GEBIM), da Faculdade de Engenharia da Universidade Federal de Juiz de Fora, ele teve início no segundo semestre de 2018 com o objetivo de suprir a necessidade do conhecimento amplo do BIM, como competência emergente no campo da AECO (Arquitetura, Engenharia, Construção e Operações), a partir da iniciativa de um grupo de discentes após conhecerem o BIM. O estudo do BIM dentro da Universidade é limitado, visto que, ele não faz parte do currículo do curso de Engenharia Civil, ficando restrito apenas a algumas iniciativas pontuais em algumas disciplinas do curso. A partir daí, estes discentes viram no GEInfra, grupo também pertencente ao Núcleo de Estudos e Projetos em Educação e Tecnologia (NETEC), a inspiração para criação do GEBIM.

Desde o início, o trabalho do GEBIM, tem-se pautado por uma perspectiva ampla, abrangendo não apenas o software de modelagem, como também uma visão do processo e da cultura de colaboração propiciada pelo BIM. A tecnologia é trazida através do software, onde a modelagem paramétrica traz a representação para a realidade. A visão do processo é essencial, pois é onde é controlado o fluxo de trabalho e as informações, que estão ligadas à estruturação do projeto. A cultura, ou também conhecida como política, tira o pensamento arcaico de linhas e leva a pensar em modelos e em novas ferramentas, onde a equipe trabalha, ao mesmo tempo, em um projeto colaborativo podendo explorar todo o ciclo de vida do projeto, desde a idealização até a demolição, uma visão de processo não contemplada pelo currículo do curso. O trabalho do GEBIM, aborda também assuntos ligados ao BIM, tais como o projeto de construção civil, o gerenciamento de projetos e a análise de riscos na construção civil onde professores do NETEC proporcionam os subsídios conceituais necessários.

O grupo conta com a tutoria do Prof. D.Sc. Mauricio L. Aguilar Molina, do NETEC, e é coordenado por um membro da equipe, escolhido através de votação. Os membros do GEBIM são escolhidos entre os discentes dos cursos de graduação da Faculdade de Engenharia e do curso de Arquitetura e Urbanismo da UFJF, através de um processo seletivo elaborado pelos próprios membros do grupo. O GEBIM tem como base o NETEC, onde a vivência de um trabalho em uma equipe interdisciplinar agrega valor ao grupo e os prepara para a realidade profissional.

A cada início de semestre o grupo se reúne para definição do projeto a ser desenvolvido. Após a definição, o grupo fica responsável por se dedicar a oito horas semanais, sendo duas horas correspondentes a uma reunião presencial, com todos os membros, e as demais, são livres onde cada membro se dedica a fazer um estudo teórico do projeto escolhido, um estudo sobre a aplicação dos conceitos do BIM através da utilização de softwares e a discussões de resultados com os integrantes do grupo.

Os membros dos grupos de estudos são voluntários, ou seja, estão ali inteiramente pelo fato de desejarem conhecimento. O grupo de estudos, diferente dos demais segmentos, deixa os membros livres para atuarem na área de seu interesse de modo que o integrante se sinta motivado a tirar o proveito desejado do que está fazendo. E, assim, o conhecimento é passado de integrante para integrante.

\subsection{Participação em eventos externos}

Durante o período os membros fazem parceria com outros segmentos de dentro e fora da Universidade, as quais, os membros têm a oportunidade de ministrar cursos, contribuindo com a comunidade acadêmica local, além de atingir um dos objetivos do grupo que é a disseminação 
do BIM. No caso de organizações com fins lucrativos, o grupo troca conhecimento por conhecimento. Neste caso, o grupo oferta o curso e, se for de interesse do grupo, a empresa oferece algum projeto para estudo, por exemplo.

\subsection{Desafios}

A pouca abordagem da gestão durante a graduação faz com que os alunos busquem este conhecimento dentro dos segmentos onde aprendem na base da tentativa e erro. Nos grupos de estudo não é diferente, o que dificulta o desenvolvimento dos mesmos. Quando o grupo se renova os novos membros têm que aprender como planejar, como organizar, como dirigir e como controlar, o que torna um desafio e, ao mesmo tempo, se já tivessem aprendido tais habilidades no início do curso, estariam utilizando o tempo disponível para se dedicarem integralmente ao desenvolvimento dos projetos.

\section{PESQUISA COM OS MEMBROS DOS GRUPOS}

Para verificar a importância dos Grupos de Estudos supracitados, foi feita uma pesquisa sobre os mesmos por meio de um formulário online. Foram realizadas as seguintes perguntas aos discentes participantes:

1) Há quanto tempo participa ou participou dos grupos de estudo do NETEC?

2) A participação nesses grupos acrescentou algo à sua formação? Explique.

3) A experiência de ter participado dos grupos abriu portas para outras atividades profissionais ou acadêmicas? Detalhe.

4) No caso de ainda estar participando de algum dos grupos, acredita que essa participação pode abrir portas para outras atividades profissionais ou acadêmicas? 5) No caso de ter saído de algum desses grupos, poderia informar qual foi o motivo?

6) Que opina da dinâmica "mais autônoma" que promovem estes grupos?

No caso dos professores, as perguntas foram duas:

1) De onde surgiu a ideia dos grupos de Estudo?

2) De que maneira pensa que este tipo de experiência ajuda na formação dos alunos?

Dentre os entrevistados dos grupos, 62,5\% participa dos grupos entre 1 e 2 anos, $25 \%$ há mais de 2 anos e 12,5\% há menos de 1 ano. Isso deve-se aos membros remanescentes e aos novos processos seletivos do grupo. Todos os participantes classificam como muito (75\%) ou bastante $(25 \%)$ o acréscimo de conhecimentos e experiências à sua formação. Muitos citaram a autonomia e o grande conhecimento agregado como pontos positivos do grupo, além de poderem ter tido experiências que não teriam caso estivessem realizando estudos individuais.

Ademais, 87,5\% dos entrevistados relataram que os grupos foram fundamentais para aquisição de novas experiências na universidade, além de já ter colaborado para a obtenção de vivências profissionais, como estágios e bolsas de treinamento profissional. Faz-se interessante enunciar que todos os membros que se desvincularam do núcleo o fizeram por terem seguido essas outras atividades advindas dos grupos ou mesmo pelo encerramento do curso.

Por fim, sobre a autonomia aos discentes promovida pelo grupo, todos citam que ela é um grande diferencial, visto que consegue trazer mais experiências e desenvolvimento de competências como liderança, disciplina e responsabilidade. Alguns poucos citam que ela, em partes, atrapalha os grupos devido a essa própria liberdade, o que pode causar um menor 
envolvimento por parte dos discentes, e, por conseguinte, o atraso de prazos.

Para os docentes orientadores entrevistados, o desenvolvimento de projetos pelos alunos contribui na aquisição de competências essenciais não contempladas no atual currículo do curso, como projeto, gestão, trabalho em equipe em rede.

\section{CONSIDERAÇÕES FINAIS}

Pode-se considerar que o modelo de grupos de estudo, é uma ótima alternativa para suprir as necessidades de novos conhecimentos, dentro de cada área, tendo em vista que, a todo momento há o surgimento de uma nova tecnologia, como o BIM, de uma nova necessidade da sociedade, como o estudo de barragens, evolução esta que a grade curricular não consegue acompanhar.

A horizontalidade de responsabilidades traz liberdade para os integrantes e com ela a responsabilidade, disciplina, autoliderança e organização que são habilidades imprescindíveis na carreira profissional. Portanto, considera-se positivo o resultado que o modelo de grupos de estudo do NETEC vem apresentando no aprendizado na graduação, devido a satisfação de atuais e ex-membros, e da abertura de oportunidades no mercado de trabalho para os mesmos.

\section{Agradecimentos}

Agradecemos à Universidade Federal de Juiz de Fora, à Faculdade de Engenharia e ao NETEC.

\section{REFERÊNCIAS}

ALERTE, Jac-ssone. PROPOSTA DE (RE) CONSTRUÇÃO DE CASAS POPULARES EM REGIME DE MUTIRÃO COMO ALTERNATIVA AO DÉFICIT HABITACIONAL DO HAITI. 2017. 106 f. Trabalho de Conclusão de Curso. Escola Politécnica, Universidade Federal do Rio de Janeiro, Rio de Janeiro, 2017.

BRASIL. Ministério da Educação. Resolução CNE/CES No. 2 de 24 de Abril de 2019. Institui as Diretrizes Curriculares Nacionais dos Cursos de Graduação em Engenharia, Diário Oficial da União No. 80, 26 de Abril de 2019, Seção 1, Pág. 43-44, Brasília, 2019

CORPORATE FINANCE REVIEW: The Importance of Soft Skills. Nova York: [si], v. 14, n. 6, jun. 2010. Mensal. Disponível em: https://search.proquest.com/openview/aa5f345bde23cacbf99574378aeef44b/1?pqorigsite=gsc holar\&cbl=46775. Acesso em: 31 maio 2020.

LEVY, P. A Inteligência Coletiva: Por uma Antropologia do Ciberespaço, Trad. Rouanet, P., São Paulo: Edições Loyola, 1998.

MORÁN, José. Mudando a educação com metodologias ativas. Disponível em: https://www.ucs.br/site/midia/arquivos/bibliografia-PGCIMA-canela.pdf. Acesso em: 31 Maio 2020.

MYERS, D. Psicologia Social, Porto Alegre: AMGH Editora Ltda., 2014. 
SOBRAL, Fernanda Ribeiro. CAMPOS, Claudinei José Gomes. Utilização de metodologia ativa no ensino e assistência de enfermagem na produção nacional: revisão integrativa. Rev Esc Enferm, USP. 2012. Disponível em: www.ee.usp.br/reeusp. Acesso em: 15 maio 2020.

UNIVERSIDADE FEDERAL DE JUIZ DE FORA. Grupo de Estudos e Práticas em BIM. Disponível em: https://www.ufjf.br/netec-feng/grupos/gebim/. Acesso em: 31 maio 2020.

UNIVERSIDADE FEDERAL DE JUIZ DE FORA. Grupo de Estudos em Grandes Obras de Infraestrutura. Disponível em: https://www.ufjf.br/netec-feng/grupos/geinfra/. Acesso em: 31 maio 2020.

UNIVERSIDADE FEDERAL DE JUIZ DE FORA. Regimento Acadêmico da Graduação. Artigo 72 e 73, de 25 de janeiro de 2016. Juiz de Fora, MG, p. 22-23. Disponível em: http://www.ufjf.br/arquitetura/files/2017/07/RegulamentoAcad\%C3\%AAmicodaGradua\%C3 \%A7\%C3\%A3o-RAG.pdf. Acesso em: 31 maio 2020.

\section{THE USE OF STUDY GROUPS FOR LEARNING IN GRADUATION}

Abstract: The new curricular guidelines advocate greater training of soft skills in Engineering courses and, among the skills to be developed, the ability to work in teams, creativity and criticism are highly recommended. In line with this guideline, at the Federal University of Juiz de Fora there is a regulation that promotes teaching activities outside the classroom, privileging more efficient learning spaces that make the student the active agent of their learning. Group work can therefore be a good model for developing the desired skills. This study presents and discusses the methodology of the group of studies that adopt a perspective of greater autonomy of students in the organization of activities. First, the context and general framework of the proposal is presented, and then the results obtained through this methodological practice are listed. Finally, the results of a qualitative research are presented to describe the high receptivity that this approach has among students and teachers.

Keywords: Teaching methodology, Study Groups, Education. 